\title{
Satisfação de usuários de aparelho de amplificação sonora individual após utilização de um site responsivo no processo de adaptação
}

\section{Satisfaction of users of hearing aids after using a responsive website}

\author{
in the adaptation process
}

\author{
Laryssa da Silva Gomes ${ }^{1}$, , Pedro de Lemos Menezes ${ }^{1,2}$ (1), Gabriella Oliveira Peixoto ${ }^{1}$ (D), \\ Natália dos Santos Pinheiro ${ }^{3}$ (D), Maria Cecília dos Santos Marques ${ }^{1}$ (D), Aline Tenório Lins Carnaúba ${ }^{1,2}$ (1), \\ Klinger Vagner Teixeira da Costa ${ }^{1,2}$ (D), Kelly Cristina Lira de Andrade ${ }^{1,2}$ (1)
}

\begin{abstract}
RESUMO
Objetivo: $\mathrm{O}$ aparelho de amplificação sonora individual propicia benefícios para indivíduos com deficiência auditiva. No entanto, ainda são observados baixos níveis de adesão ao tratamento, devido às dificuldades no processo de adaptação. Neste sentido, destaca-se o uso de sites ou aplicativos com os objetivos de auxiliar no processo de orientação aos usuários e de avaliar a satisfação de usuários de aparelho de amplificação sonora individual, após a utilização de um site responsivo desenvolvido para auxiliar no processo de adaptação. Métodos: Estudo quantitativo analítico experimental, ensaio clínico não randomizado, com 20 participantes, divididos em dois grupos. O grupo estudo fez uso do site responsivo como ferramenta de auxílio na adaptação, além de receber as orientações em seu formato tradicional. O grupo controle foi orientado somente pelo formato tradicional já existente no serviço. Após um mês, os participantes responderam a um questionário de avaliação de satisfação e também foi realizada a análise da ferramenta datalogging. Resultados: O site responsivo contou com orientações sobre manutenção e dicas de uso do aparelho de amplificação sonora individual, permitindo fácil acesso às principais orientações ao usuário inexperiente. A análise dos dados demonstrou respostas mais positivas ao questionário pelo grupo estudo, bem como maior tempo de uso diário dos aparelhos de amplificação sonora individuais. Conclusão: o grupo que utilizou o site responsivo como ferramenta complementar ao processo de adaptação do aparelho de amplificação sonora individual mostrou, a partir da avaliação da satisfação e dos dados da ferramenta datalogging, maior satisfação e maior tempo de uso diário do aparelho.
\end{abstract}

Palavras-chave: Perda auditiva; Auxiliares de audição; Tecnologia; Sistema Único de Saúde; Inquéritos e questionários

\begin{abstract}
Purpose: The hearing aid provides benefits for individuals with hearing impairment. However, low levels of treatment adherence are still observed due to difficulties in the adaptation process. In this sense, the use of websites or applications stands out in order to assist in the process of orienting users. To evaluate the satisfaction of users of hearing aids after using a responsive website designed to assist in the adaptation process. Methods: Quantitative analytical experimental study non-randomized clinical trial whit 20 participants, divided into two groups. The study group made use of the responsive website as an aid tool in adapting, in addition to also receiving the guidelines in their traditional format. The control group was guided only with the traditional format already existing in the service. After one month, the participants answered a satisfaction assessment questionnaire, as well as an analysis of the datalogging. Results: The responsive website had guidance on maintenance and tips on using the individual hearing aid, allowing easy access to the main guidelines for inexperienced users. The analysis of the data showed more positive responses to the questionnaire by the study group, as well as a longer time of daily use of hearing aids. Conclusion: The group that used the responsive website as a complementary tool to the adaptation process of the hearing aid showed, from the assessment of satisfaction and data from the datalogging tool, greater satisfaction and a longer time of daily use of the hearing aid.
\end{abstract}

Keywords: Hearing loss; Hearing aids; Technology; Unified health system; Surveys and questionnaires

\footnotetext{
Trabalho realizado no Centro Especializado em Reabilitação III, Universidade Estadual de Ciências da Saúde de Alagoas - UNCISAL - Maceió (AL), Brasil. ${ }^{1}$ Laboratório de Audição e Tecnologia - LATEC, Universidade Estadual de Ciências da Saúde de Alagoas - UNCISAL - Maceió (AL), Brasil.

${ }^{2}$ Centro Universitário CESMAC - Maceió (AL), Brasil.

${ }^{3}$ Programa de Pós-graduação em Saúde da Comunicação Humana, Universidade Federal de Pernambuco - UFPE - Recife (PE), Brasil.

Conflito de interesses: Não.

Contribuição dos autores: LSG foi responsável pela concepção e delineamento do estudo, coleta, análise e interpretação dos dados; PLM foi responsável pela concepção e delineamento do estudo, coleta, análise e interpretação dos dados e aprovação final da versão a ser publicada; GOP foi responsável pela concepção e delineamento do estudo, coleta, análise e interpretação dos dados; NSP foi responsável pela concepção e delineamento do estudo, coleta, análise e interpretação dos dados e aprovação final da versão a ser publicada; MCSM foi responsável pela concepção e delineamento do estudo, coleta, análise e interpretação dos dados; ATLC foi responsável pela redação ou revisão do artigo de forma intelectualmente importante; KVTC foi responsável pela redação ou revisão do artigo de forma intelectualmente importante; KCLA foi responsável pela concepção e delineamento do estudo, coleta, análise e interpretação dos dados, redação ou revisão do artigo de forma intelectualmente importante e aprovação final da versão a ser publicada.
}

Financiamento: Nada a declarar.

Autor correspondente: Kelly Cristina Lira de Andrade. E-mail: kellyclandrade@gmail.com

Recebido: Junho 08, 2020; Aceito: Novembro 30, 2020 


\section{INTRODUÇÃO}

A audição é fundamental nas situações de interação social, quando a comunicação ocorre por meio oral. Contudo, a perda auditiva não afeta somente a capacidade do indivíduo de compreender adequadamente as informações sonoras, mas, principalmente, o modo de se relacionar com seu meio e cultura, podendo provocar consequências biológicas, psicológicas e sociais $^{(1-3)}$.

Desse modo, é crucial que o diagnóstico da perda auditiva e a intervenção sejam realizados precocemente. O processo de reabilitação, em muitas situações, ocorre com a indicação, seleção e adaptação do aparelho de amplificação sonora individual (AASI) ${ }^{(4,5)}$.

Os serviços de saúde auditiva habilitados pelo Ministério da Saúde devem oferecer um processo de intervenção que contemple o diagnóstico audiológico, a seleção e adaptação do AASI adequado às características e necessidades acústicas do indivíduo, o acompanhamento audiológico e a terapia fonoaudiológica para o desenvolvimento das suas habilidades auditivas e de linguagem ${ }^{(6)}$.

Alguns usuários e suas famílias envolvem-se rapidamente no processo de adaptação dos AASI, o que facilita a evolução. No entanto, grande parte dos usuários, embora frequente regularmente as terapias, não utiliza os seus AASI em outras situações cotidianas, um dos motivos pelo qual é necessário que o serviço ofereça ações que auxiliem a adesão ao tratamento ${ }^{(5)}$.

Estudo $^{(3)}$ demonstrou que, mesmo havendo grande ocorrência de perda auditiva na população idosa, apenas $20 \%$ desses indivíduos adquirem AASI. Além disso, cerca de $30 \%$ dos usuários mencionaram insatisfação com seus AASI e aproximadamente $16 \%$ relataram nunca ter utilizado esses dispositivos de forma efetiva ${ }^{(3)}$.

Diante disso e frente à possibilidade do uso da tecnologia a favor de suas aplicações na saúde, ressaltam-se os benefícios do uso de sites e aplicativos para as intervenções em saúde e aperfeiçoamento das tomadas de decisões clínicas $^{(7)}$.

No Brasil, 93,2\% dos domicílios têm usuários de smartphones, um número expressivo e que se deve à facilidade de compra, usabilidade e facilidade de conexão com o mundo virtual. Esses fatos tem implicação direta na sociedade e garantem um elevado potencial de uso na área de educação, saúde e cuidado integral ${ }^{(8)}$.

Um extenso trabalho de revisão do uso de ferramentas móveis elencou algumas das características que favorecem o uso de aplicações no contexto de saúde, destacando-se acessibilidade, mobilidade, baixo custo, capacidade contínua de transmissão de dados, geolocalização e capacidade multimídia ${ }^{(9)}$.

Um site responsivo é aquele que se adapta a qualquer dispositivo móvel e, pelo fato de haver um número expressivo de pessoas que possuem smartphones, conforme mencionado anteriormente, a sua utilização pode auxiliar o acesso às orientações sobre o uso adequado do AASI, caracterizando-se como uma possibilidade bastante viável.

Com base nos importantes benefícios propiciados pelo uso do AASI por indivíduos com deficiência auditiva e nas dificuldades de adesão ao tratamento, o objetivo deste estudo foi avaliar a satisfação desses usuários após a utilização de um site responsivo, desenvolvido para auxiliar no processo de adaptação do dispositivo.

\section{MÉTODO}

Trata-se de um estudo de caráter quantitativo analítico experimental, ensaio clínico não randomizado, realizado no período de julho a setembro de 2018 com indivíduos de ambos os sexos, em uma clínica escola que concede AASI pelo Sistema Único de Saúde (SUS), pertencente à Universidade Estadual de Ciências da Saúde de Alagoas (UNCISAL). O estudo foi aprovado pelo Comitê de Ética em Pesquisa (CEP) da instituição, sob o CAAE número 79991317.0.0000.5011 e parecer número 2.396.457.

\section{Etapa 1 - Desenvolvimento do site responsivo}

O site foi desenvolvido com o uso da linguagem de programação PHP (Hypertext Preprocessor) e sistema de gerenciador de conteúdo Wordpress. O registro do software no Instituto Nacional da Propriedade Industrial (INPI) foi expedido no dia 30/10/2018 (processo de $\mathrm{N}^{\circ}$ : BR512018051938-5).

A produção do site responsivo, registrado no endereço www. aasi.com.br, baseou-se nos seguintes critérios: 1) layout adaptado para o perfil do público-alvo, considerando-se a experiência do usuário (user experience); 2) design com o propósito de facilitar a localização das informações, legibilidade e leiturabilidade; 3) conteúdo textual objetivo e com linguagem simples; 4) vídeos curtos, claros e autoexplicativos.

A tela inicial do site exibe dois links principais: Manutenção e Dicas (Figura 1). Além disso, há um terceiro link com a nomenclatura CER III - UNCISAL (que significa Centro Especializado em Reabilitação III - UNCISAL). Conteúdo de cada link:

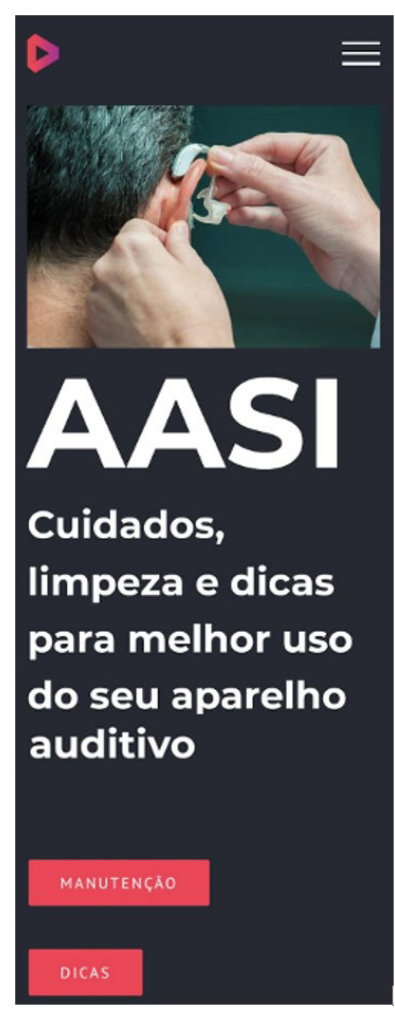

Figura 1. Layout do site desenvolvido (www.aasi.com.br) 
1. Manutenção - seis tópicos que abordam conteúdos necessários para a boa manutenção do AASI: limpeza do aparelho auditivo com molde; limpeza do aparelho auditivo com tubo fino; encaixe correto do molde; como trocar as pilhas; bombinha de ar e desumidificador;

2. Dicas - oito tópicos que abordam conteúdos com dicas para o uso do AASI: cuidados com o aparelho auditivo; qual o lado correto do aparelho auditivo?; como encaixar o aparelho auditivo com molde na orelha; como encaixar o aparelho auditivo com tubo fino na orelha; pilhas; como usar o telefone com o aparelho auditivo; evitar uso de cotonetes e dicas rápidas;

3. CER III - UNCISAL - explicação breve sobre o serviço e telefone de contato para marcação de consultas.

Os textos, fotos e vídeos permitem fácil acesso às principais orientações ao usuário inexperiente de AASI.

\section{Etapa 2 - Avaliação da satisfação do usuário de aparelho de amplificação sonora individual após a utilização do site responsivo}

A amostra foi composta por 20 participantes, sendo 14 do sexo feminino e seis do sexo masculino, selecionados por conveniência. A faixa etária dos participantes variou de 38 a 69 anos e todos assinaram o Termo de Consentimento Livre e Esclarecido (TCLE), respeitando as diretrizes e normas regulamentadoras de pesquisa em seres humanos.

No grupo controle (GC), foram incluídos indivíduos alfabetizados, com idades entre 25 e 70 anos; com perdas auditivas bilaterais de graus leve, moderado, moderadamente severo e severo; inexperientes para o uso de AASI; com indicação do otorrinolaringologista para adaptação de AASI.

No grupo de estudo (GE), foram incluídos indivíduos alfabetizados, com idades entre 25 e 70 anos; com perdas auditivas bilaterais de graus leve, moderado, moderadamente severo e severo; inexperientes para o uso de AASI; com indicação do otorrinolaringologista para o uso do AASI. Além disso, os participantes precisavam ser usuários de dispositivos móveis.

Com o objetivo de suprimir variáveis que pudessem influenciar nos resultados quanto ao tempo de uso do AASI e a satisfação desses usuários, foram excluídos do estudo indivíduos que apresentassem perda auditiva de grau profundo, qualquer alteração neurológica ou psiquiátrica, infecção de orelha média e queixa de zumbido. Para os dois últimos critérios, o objetivo foi, respectivamente, evitar o não uso do AASI por motivos de infecção de orelha média e homogeneizar os grupos quanto aos recursos dos AASI, uma vez que o uso de técnicas para o tratamento do zumbido poderia representar um viés de pesquisa.

Para a etapa de avaliação do site responsivo, com base nos dados dos prontuários e nos critérios de inclusão e exclusão estabelecidos, os possíveis participantes da pesquisa foram selecionados e convidados. Após o aceite para participação, foram explicadas, oralmente, as condutas a serem realizadas e, em seguida, o TCLE foi assinado. Posteriormente, foram realizados os seguintes procedimentos:

1. O GE foi composto por dez indivíduos que receberam orientações em formato tradicional, no ato da concessão do AASI e fizeram uso do site responsivo por meio de smartphones. O formato tradicional consistiu em orientações descritas em um folder impresso em papel A4, bem como de explicações demonstradas na prática, pelo fonoaudiólogo do serviço. As informações e dúvidas sobre o site responsivo também foram esclarecidas no dia da adaptação do AASI. O site responsivo é um recurso tecnológico educativo, que se adapta a qualquer dispositivo móvel, como celulares ou tablets. Dispõe de orientações textuais, fotos e vídeos sobre o uso, manuseio, troca de pilhas, higiene e limpeza do AASI;

2. O GC foi composto por dez indivíduos que não utilizaram o site responsivo como ferramenta de auxílio na adaptação do AASI, sendo estes orientados somente com o formato tradicional já existente no serviço;

3. Após um mês da concessão do AASI, os participantes dos dois grupos retornaram ao serviço para a primeira sessão de revisão e preencheram um questionário de avaliação da satisfação, o Satisfaction With Amplification in Daily Life (SADL) (Anexo 1) ${ }^{(10)}$. O SADL é um questionário útil para a aplicação clínica $^{(11)}$ e se caracteriza por ser um instrumento que avalia a medida de satisfação do usuário com o uso do AASI na vida diária. É composto por 15 questões, divididas em quatro subescalas: 1) efeitos positivos (seis itens associados com os benefícios acústico e psicológico); 2) serviços e custos (três itens associados com a competência profissional, o preço do produto e o número de consertos); 3 ) fatores negativos (três itens relacionados com a amplificação de ruído ambiental, a presença de realimentação e o uso de telefone); 4) imagem pessoal (três itens relacionados com fatores estéticos e o estigma do uso do AASI).

Os participantes responderam cada questão, escolhendo uma das possíveis respostas: nada, um pouco, de alguma forma, mediamente, consideravelmente, muito e muitíssimo. Para 11 questões, "muitíssimo" indica total satisfação e é pontuada com o valor 7, enquanto "nada" indica total insatisfação e é pontuada com o valor 1 (questões $1,3,5,6,8,9,10,11,12,14$, 15). Porém, as demais questões são invertidas e "muitíssimo" indica total insatisfação, sendo pontuada com o valor 1 e "nada" indica total satisfação, sendo pontuada com o valor $7^{(12)}$.

Os escores de satisfação foram calculados por média aritmética, por meio de normas somáticas dos pontos nos itens do questionário. Considerou-se "insatisfeito" aquele com escore abaixo do valor normativo relativo a $20^{\circ}$ percentil, como "satisfeito" aquele com escore entre o $20^{\circ}$ e o $80^{\circ}$ percentil e como "muito satisfeito" aquele com escore acima de $80^{\circ}$ percentil ${ }^{(13)}$. Como a pesquisa foi realizada em um centro de reabilitação que fornece os AASI pelo SUS, a pergunta de número 14 não foi pertinente e, por isso, desconsiderada do estudo.

4. Nesta mesma sessão, para os dois grupos estudados, foi realizada a análise da ferramenta de registro de dados (datalogging), ativada no dia da adaptação, com o objetivo de verificar o número de horas diárias de uso do AASI;

5. Por fim, as respostas ao questionário foram comparadas aos dados da ferramenta datalogging. 


\section{Análise estatística}

A análise estatística foi realizada por meio do aplicativo BioEstat, versão 5.0 para Windows. Para a descrição dos dados, foi utilizada apresentação tabular e gráfica das médias e dos desvios padrão. Inicialmente foi realizada uma avaliação da amostra, para observar sua aderência à distribuição normal, por meio do teste de Lilliefors. Para avaliação das perguntas do questionário SADL, foi utilizado o teste não paramétrico de Mann-Whitney para amostras independentes. Em seguida, para a comparação entre os grupos quanto aos dados do datalogging, foi utilizado o teste $t$ de Student. As diferenças foram consideradas significativas para os valores de p menores que 0,05 e o valor de beta admitido foi de 0,1 .

\section{RESULTADOS}

A amostra foi composta por 20 participantes, sendo 14 $(70 \%)$ do sexo feminino e $6(30 \%)$ do sexo masculino. A faixa etária dos participantes variou de 38 a 69 anos (média de idade de 56,05 anos e desvio padrão de 10,45 anos) e todos eram usuários de smartphones. A distribuição por sexo, faixa etária, média de idade e desvio padrão, por grupo, encontra-se descrita na Tabela 1.

Quanto ao nível socioeconômico, a distribuição da amostra por grupo apresentou-se da seguinte forma: GE - $20 \%$ corresponderam à renda mensal familiar de, aproximadamente, 6 salários mínimos e $80 \%$, à renda mensal familiar de, aproximadamente, 2 salários mínimos; GC - 30\% corresponderam à renda mensal familiar de, aproximadamente, 6 salários mínimos e $70 \%$, à renda mensal familiar de, aproximadamente, 2 salários mínimos.
Quanto à escolaridade, a distribuição se apresentou da seguinte forma: GE - $40 \%$ com o ensino médio completo e $60 \%$ com o ensino fundamental completo; $\mathrm{GC}-30 \%$ com o ensino médio completo e $70 \%$ com o ensino fundamental completo.

Para as duas variáveis relatadas acima, por meio do teste estatístico Qui-quadrado, não foram observadas diferenças estatísticas entre os dois grupos $(\mathrm{p}=0,606$ para renda familiar e $\mathrm{p}=0,639$ para escolaridade).

Todos os participantes do estudo foram adaptados com AASI retroauricular, conforme disponibilidade do serviço (Figura 2).

Foram encontradas perdas auditivas de diferentes tipos e graus para os dois grupos estudados (Tabela 2).

Sobre o estudo do tráfego do site durante o período de coleta de dados, contabilizou-se um total de 83 acessos. As páginas mais acessadas, desconsiderando-se a página inicial, foram

Tabela 2. Caracterização das perdas auditivas dos participantes do estudo, quanto aos tipos e graus

\begin{tabular}{lll}
\hline \multicolumn{1}{c}{ Grupo Controle } & $\mathbf{n}$ & $\%$ \\
\hline PASN & 2 & 20 \\
PASN leve & 2 & 20 \\
PASN moderada & 3 & 30 \\
PASN moderadamente severa & 1 & 10 \\
PAM leve & 2 & 20 \\
\hline \multicolumn{1}{c}{ Grupo Estudo } & $\mathbf{n}$ & $\%$ \\
\hline PASN & 3 & 30 \\
PASN leve & 1 & 10 \\
PASN moderada & 2 & 20 \\
PASN moderada (OD)/severa (OE) & 2 & 20 \\
PAM moderadamente severa & 1 & 10 \\
PAM moderada (OD)/PASN (OE) & 1 & 10 \\
\hline
\end{tabular}

Legenda: $n=$ número de participantes $\%=$ percentual; PASN $=$ perda auditiva sensorioneural; $\mathrm{PAM}=$ perda auditiva mista; $\mathrm{OD}=$ orelha direita; $\mathrm{OE}=$ orelha esquerda

Marcas e aparelhos de amplificação sonora individuais adaptados

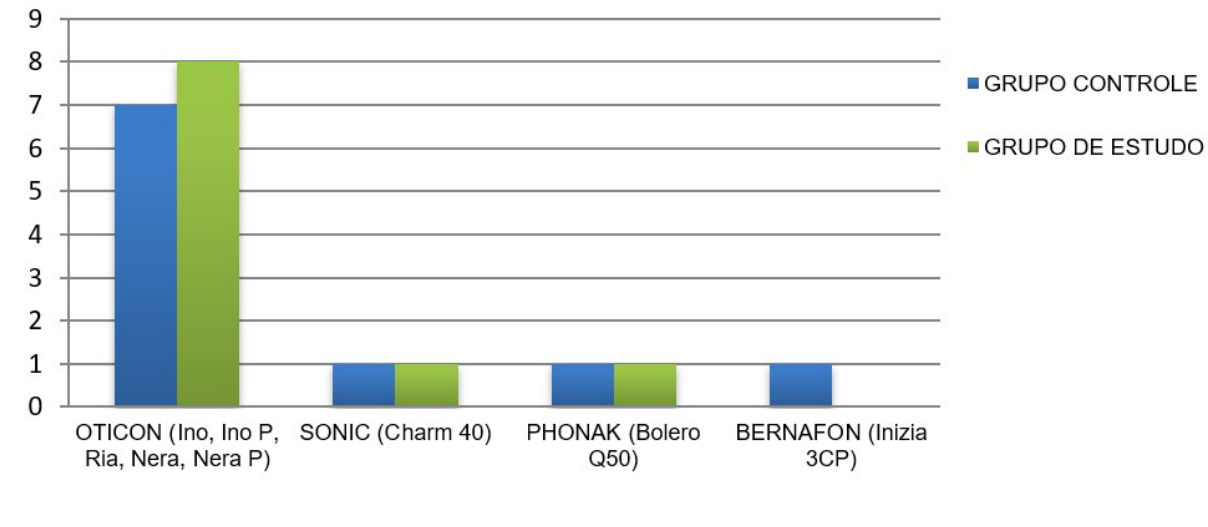

Figura 2. Marcas e aparelhos de amplificação sonora individuais adaptados, por grupo estudado Fonte: Dados da pesquisa

Tabela 1. Distribuição por sexo, faixa etária, média de idade e desvio padrão, por grupo estudado

\begin{tabular}{ccccccc}
\hline Grupo & $\begin{array}{c}\text { Sexo masculino } \\
(\%)\end{array}$ & $\begin{array}{c}\text { Sexo feminino } \\
(\%)\end{array}$ & $\begin{array}{c}\text { Faixa etária } \\
\text { (anos) }\end{array}$ & $\begin{array}{c}\text { Média de idade } \\
\text { (anos) }\end{array}$ & $\begin{array}{c}\text { Desvio padrão } \\
\text { (anos) }\end{array}$ & $\begin{array}{c}\mathbf{n} \\
\text { GC }\end{array}$ \\
GE & 40 & 60 & $40-69$ & 54,3 & 11,02 & 10 \\
10,12 & 10 \\
\hline
\end{tabular}

Legenda: $\%$ = percentual; GC = grupo controle; GE = grupo de estudo; $n$ = número de participantes. Fonte: Dados da pesquisa 
nesta ordem: "Qual o lado correto do aparelho auditivo?", "Pilhas", "Como trocar as pilhas" e "Como usar o telefone com o aparelho auditivo".

A partir dos valores de referência preestabelecidos, determinouse o perfil dos participantes, de acordo com o escore global de satisfação para o SADL. O GC apresentou média de $23 \%$ para "muito satisfeitos" e 6,1\% para "insatisfeitos". O GE, por sua vez, apresentou média de $62,7 \%$ para "muito satisfeitos" e 2,2\% para "insatisfeitos".

Para a comparação das respostas do questionário SADL por grupo, foi utilizado o teste não paramétrico de Mann Whitney para amostras independentes (Tabela 3).

Para a avaliação do datalogging por grupo estudado, o teste de Lilliefors demonstrou distribuição normal da amostra. Desta forma, a análise foi realizada por meio do teste $\mathrm{t}$ de Student, com valor de $\mathrm{p}<0,001$. A média de horas por dia foi de 2,77 horas para o grupo controle (desvio padrão de 2,07 horas) e de 7,92 horas para o grupo de estudo (desvio padrão de 1,8 horas).
A Figura 3 representa a quantidade de horas de uso do AASI por dia, por participante, de acordo com o grupo estudado.

\section{DISCUSSÃO}

Fornecer subsídios para a indicação de recurso educativo favorável e de melhor método de acompanhamento na reabilitação auditiva caracteriza-se como uma medida importante e urgente, uma vez que propiciará a redução da evasão e insatisfação dos usuários de AASI. Além disso, identificar as causas e desenvolver estratégias para atingir a eficácia nesta intervenção é fundamental ${ }^{(13)}$.

No presente estudo, que teve como temática o uso de um site com orientações acerca do uso do AASI, houve maior prevalência de participantes do sexo feminino. A média de idade, independente do grupo, representou uma população

Tabela 3. Quantidade de pessoas que responderam determinadas alternativas por grupo estudado

\begin{tabular}{|c|c|c|c|c|c|c|c|c|c|}
\hline Questão & Grupo & Nada & Um pouco & $\begin{array}{c}\text { De alguma } \\
\text { forma }\end{array}$ & Mediamente & Consideravelmente & Muito & Muitíssimo & Valor de $p$ \\
\hline \multirow[t]{2}{*}{ Q1 } & $\mathrm{GC}$ & 1 & 0 & 1 & 3 & 0 & 1 & 4 & $0,03^{*}$ \\
\hline & GE & 0 & 0 & 0 & 0 & 0 & 1 & 9 & \\
\hline \multirow[t]{2}{*}{ Q2 } & $\mathrm{GC}$ & 7 & 0 & 1 & 2 & 0 & 0 & 0 & 0,90 \\
\hline & GE & 6 & 2 & 1 & 1 & 0 & 0 & 0 & \\
\hline \multirow[t]{2}{*}{ Q3 } & $\mathrm{GC}$ & 2 & 0 & 0 & 3 & 0 & 3 & 2 & $0,004^{\star}$ \\
\hline & GE & 0 & 0 & 0 & 0 & 0 & 1 & 9 & \\
\hline \multirow[t]{2}{*}{ Q4 } & $\mathrm{GC}$ & 2 & 0 & 1 & 0 & 4 & 1 & 1 & $0,04^{*}$ \\
\hline & GE & 8 & 0 & 0 & 0 & 2 & 0 & 0 & \\
\hline \multirow[t]{2}{*}{ Q5 } & $\mathrm{GC}$ & 1 & 0 & 0 & 0 & 5 & 0 & 4 & $0,36^{*}$ \\
\hline & GE & 0 & 0 & 0 & 1 & 2 & 1 & 6 & \\
\hline \multirow[t]{2}{*}{ Q6 } & $\mathrm{GC}$ & 0 & 1 & 1 & 0 & 4 & 0 & 4 & $0,02^{*}$ \\
\hline & GE & 0 & 0 & 0 & 0 & 0 & 0 & 10 & \\
\hline \multirow[t]{2}{*}{ Q7 } & $\mathrm{GC}$ & 1 & 0 & 1 & 1 & 2 & 4 & 1 & $0,001^{*}$ \\
\hline & GE & 8 & 0 & 0 & 2 & 0 & 0 & 0 & \\
\hline \multirow[t]{2}{*}{ Q8 } & $\mathrm{GC}$ & 0 & 1 & 1 & 1 & 2 & 2 & 3 & $0,00^{*}$ \\
\hline & $\mathrm{GE}$ & 0 & 0 & 0 & 0 & 0 & 0 & 10 & \\
\hline \multirow[t]{2}{*}{ Q9 } & $\mathrm{GC}$ & 0 & 0 & 0 & 1 & 4 & 2 & 3 & $0,01^{*}$ \\
\hline & $\mathrm{GE}$ & 0 & 0 & 0 & 0 & 0 & 1 & 9 & \\
\hline \multirow[t]{2}{*}{ Q10 } & $\mathrm{GC}$ & 2 & 0 & 0 & 0 & 0 & 2 & 6 & 0,54 \\
\hline & $\mathrm{GE}$ & 0 & 0 & 0 & 1 & 1 & 1 & 7 & \\
\hline \multirow[t]{2}{*}{ Q11 } & $\mathrm{GC}$ & 1 & 0 & 1 & 2 & 1 & 4 & 1 & $0,02^{*}$ \\
\hline & GE & 0 & 0 & 0 & 0 & 1 & 4 & 5 & \\
\hline \multirow[t]{2}{*}{ Q12 } & $\mathrm{GC}$ & 0 & 0 & 1 & 2 & 0 & 3 & 4 & $0,02^{*}$ \\
\hline & $\mathrm{GE}$ & 0 & 0 & 0 & 0 & 0 & 0 & 10 & \\
\hline \multirow[t]{2}{*}{ Q13 } & $\mathrm{GC}$ & 2 & 0 & 1 & 3 & 4 & 0 & 0 & $0,002^{*}$ \\
\hline & GE & 10 & 0 & 0 & 0 & 0 & 0 & 0 & \\
\hline \multirow[t]{2}{*}{ Q14 } & $\mathrm{GC}$ & - & - & - & - & - & - & - & - \\
\hline & GE & - & - & - & - & - & - & - & \\
\hline \multirow[t]{2}{*}{ Q15 } & $\mathrm{GC}$ & 3 & 0 & 0 & 1 & 2 & 1 & 3 & $0,008^{*}$ \\
\hline & GE & 0 & 0 & 0 & 0 & 0 & 0 & 10 & \\
\hline
\end{tabular}

\footnotetext{
Teste Mann Whitney; *Valor estatisticamente significante $(p<0,05)$
}

Legenda: $\mathrm{GC}$ = grupo controle; GE = grupo de estudo; Q1 = o seu aparelho auditivo lhe ajuda a entender as pessoas com as quais você fala, comparado a época que não usava aparelho auditivo?; Q2 = você fica frustrado quando seu aparelho auditivo capta sons que lhe impedem de ouvir o que você quer?; Q3 = você está convencido de que a obtenção de seu aparelho auditivo fazia parte dos seus maiores interesses?; Q4 = você acha que as pessoas percebem mais sua perda auditiva quando você usa os aparelhos?; Q5 = o seu aparelho auditivo reduz o número de vezes que você tem que pedir para as pessoas repetirem?; Q6 = você acha que vale a pena usar o aparelho auditivo?; Q7 = você se sente incomodado quando necessita aumentar o volume e ocorre microfonia?; Q8 = você está contente com a aparência do seu aparelho auditivo?; Q9 = o uso do seu aparelho auditivo melhora sua autoconfiança?; Q10 = o som do seu aparelho auditivo é um som natural?; Q11 = o seu aparelho auditivo é útil na MAIORIA dos telefones sem amplificador ou caixas de som?; Q12 = a pessoa que lhe forneceu o aparelho auditivo era competente?; Q13 = você acha que usar aparelho lhe faz parecer menos capacitado?; Q14 = o custo do seu aparelho auditivo parece razoável para você?; Q15 = você está satisfeito com a frequência com a qual o seu aparelho auditivo precisa de reparos? Fonte: Dados da pesquisa 
Dados do Datalogging

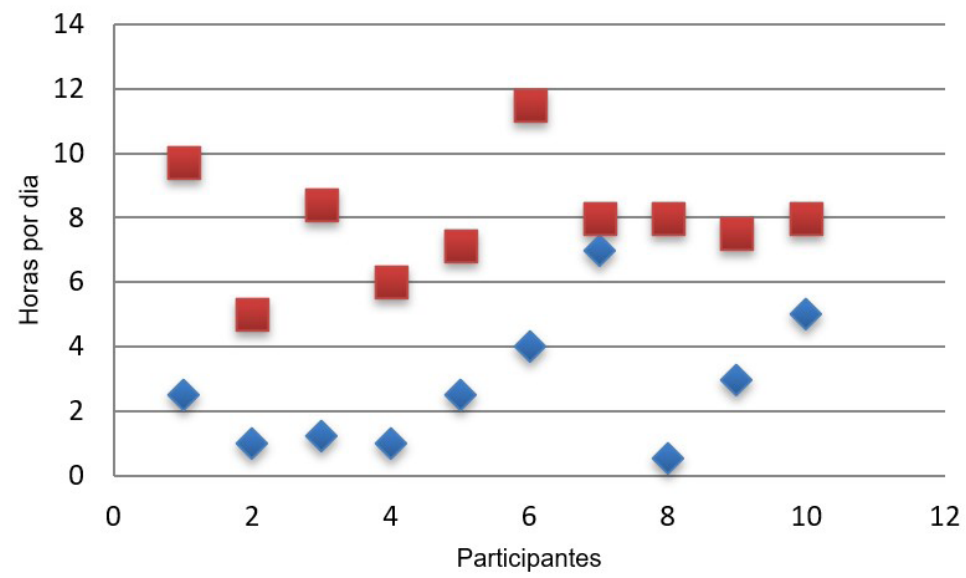

- GRUPO CONTROLE

- GRUPO DE ESTUDO

Figura 3. Quantidade de horas de uso dos aparelhos de amplificação sonora individuais por dia, de acordo com o grupo estudado Fonte: Dados da pesquisa

que, em geral, é usuária de smartphones, o que foi comprovado durante a pesquisa.

Sobre as perdas auditivas descritas na Tabela 2, verificou-se maior prevalência do tipo sensorioneural, dado similar ao de um estudo que teve como objetivo conhecer a satisfação do uso de AASI entre usuários assistidos por um serviço de saúde auditiva, identificando os fatores associados e a percepção sobre a assistência ${ }^{(13)}$.

A partir dos achados, foram observadas respostas positivas quanto ao uso do AASI após a utilização do site responsivo, bem como maior tempo de uso no GE, resultado que pode ser explicado pela utilização cada vez maior de dispositivos móveis, que permitem a troca de mensagens, a leitura de notícias, a realização de diversas outras tarefas diárias, inclusive, de informações referentes às orientações de saúde ${ }^{(9)}$.

Sendo assim, a criação de um site responsivo com acesso por dispositivos móveis, se propôs a auxiliar e beneficiar o processo de adaptação de usuários, no que se refere às orientações de uso, uma vez que estas são fornecidas, em sua maioria, de forma oral ou escrita, no dia da adaptação. Em muitos casos, os usuários desprezam as informações disponibilizadas de forma tradicional e, quando necessitam, de fato, realizar manutenções ou ajustes no AASI, apresentam dificuldades em recordá-las, situação que acarreta a degradação mais rápida do dispositivo e/ou até o abandono deste.

Pesquisa $^{(14)}$ revelou que os AASIs retroauriculares e de categoria tecnológica A (modelos mais distribuídos no SUS e utilizados na presente pesquisa), são os que apresentam maior número de falhas técnicas, mais encontradas nos amplificadores e nos microfones. Os autores concluíram que, em $50 \%$ dos casos analisados, a falha técnica ocorreu em até 16 meses pósadaptação do AASI, ainda na garantia.

É importante ressaltar que não é comum que uma grande quantidade de falhas técnicas ocorra no período de garantia. $\mathrm{O}$ resultado da pesquisa mencionada pode ter ocorrido pela falta ou pouca orientação com relação à manutenção/limpeza, introdução de água e poeira no AASI e/ou pilhas esquecidas dentro do dispositivo, o que causa oxidação e danos ao circuito. Dessa forma, a utilização de ilustrações e vídeos explicativos no site responsivo em questão se propôs a diminuir essa estatística.
A avaliação da satisfação dos usuários de AASI pode ser realizada a partir de diversas perspectivas e os questionários representam uma prática comum e eficaz na rotina clínica. O questionário SADL aborda temas que avaliam a satisfação dos usuários com relação ao AASI, apresentando questões diretamente ligadas a itens positivos (associados aos benefícios acústico e psicológico, serviços e custos, competência profissional, preço do produto e número de consertos) assim como itens ligados a fatores negativos (relacionados com a amplificação de ruído ambiental, presença de realimentação e uso de telefone) e itens ligados à imagem pessoal (fatores estéticos e estigma do uso do AASI) ${ }^{(10)}$.

O SADL, inclusive, tem demostrado ser um instrumento útil para avaliar a satisfação e qualidade de vida em usuários de implante auditivo de tronco cerebral, em associação ao Questionnaire for Measuring Health-Related Quality of Life in Children and Adolescents (KINDLR) para crianças e adolescentes, pais e/ou cuidadores e ao questionário World Health Organization Quality of Life - Bref(WHOQOL-BREF), questionário abreviado do WHOQOL-100, para os participantes adultos, conforme pesquisa realizada com 19 usuários de implante auditivo de tronco cerebral ${ }^{(15)}$.

A comparação dos dois grupos estudados na presente pesquisa, a partir das respostas ao SADL, mostrou respostas mais positivas para o GE, isto é, para o grupo que utilizou o site responsivo como ferramenta de auxílio à adaptação do AASI, quando comparado ao GC.

Tendo em vista que alguns pontos do questionário estão ligados à saúde psicológica do indivíduo e à acústica do AASI e, por isso, não possuem relação direta com o site responsivo, questões como a de número 2 , em que se avalia a captação dos sons que impedem a escuta de determinados sons alvo, não demonstraram significância estatística, achado possivelmente explicado pelo fato de a referida questão não ser influenciada pelo uso do site, mas pela tecnologia e recursos oferecidos pelos AASI.

Referente à questão de número 3 , em que se pergunta ao usuário se a obtenção do AASI fazia parte dos seus maiores interesses, houve significância estatística para o GE, o que demonstra valorização e, em última análise, melhor adaptação ao AASI. 
Em relação à pergunta de número 10 , em que se questiona se o AASI proporciona um som natural, observou-se que não houve significância estatística. Esse dado demonstra, mais uma vez, a não interferência do uso do site responsivo, mas a qualidade acústica dos dispositivos e as características específicas de cada AASI, a depender de sua tecnologia e recursos disponíveis.

Todas as demais questões, sejam referentes à capacidade de compreensão da fala, aos ganhos alcançados, à satisfação com o uso do AASI, à competência do profissional que realizou o atendimento ou à percepção do paciente se o uso do AASI o fazia parecer menos capacitado, apresentaram diferença estatística entre os dois grupos estudados, o que demonstrou importante contribuição do uso do site responsivo pelos usuários, durante a adaptação do AASI.

O presente estudo identificou $6 \%$ de usuários insatisfeitos com o AASI no grupo que não fez uso do site responsivo, dado que concorda com estudo que utilizou o SADL para avaliar a satisfação de 180 usuários de AASI e identificou 3,9\% de usuários insatisfeitos. Embora os autores tenham apontado $48,9 \%$ de usuários muito satisfeitos, relataram que esse fator foi constatado em usuários de AASI intra-auriculares, modelo que difere desta pesquisa ${ }^{(16)}$.

Sobre a análise da ferramenta datalogging, evidenciou-se diferença significativa para o GE, em que o tempo de uso diário do AASI pelos indivíduos que utilizaram o site responsivo foi, praticamente, três vezes maior que o tempo de utilização do AASI pelo GC, conforme mostra a Figura 3. O resultado sugere que novas estratégias de orientações e acompanhamento devem ser desenvolvidas e aplicadas, com o objetivo de promover a melhor adaptação ao AASI, considerando o público-alvo, suas limitações, possibilidades e preferências.

$\mathrm{O}$ datalogging demonstra ser uma ferramenta determinante, especialmente quando suas informações são relacionadas ao questionário SADL, uma vez que os dados resultaram em maior média do tempo de uso diário do AASI pelos indivíduos do GE, o que caracteriza uma avaliação positiva do site responsivo desenvolvido ${ }^{(17,18)}$.

Uma das limitações consideradas neste estudo foi o perfil do usuário atendido no serviço onde a pesquisa foi realizada, que é, em maior parte, representado por idosos e pessoas com perfil socioeconômico baixo. Desta forma, estruturar um grupo que possuísse e utilizasse dispositivos móveis e os recursos tecnológicos oferecidos representou um desafio. Contudo, a limitação não impossibilitou achados significativos e importantes, comprovados pelos resultados apresentados.

Sugere-se, portanto, que estudos similares sejam desenvolvidos com uma amostra maior e de diferentes idades, inclusive com a continuidade da atenção na adequação de recursos para cada faixa etária, sobretudo para a população infantil.

A combinação entre tecnologia e saúde auditiva pode contribuir de maneira significativa para a melhor adaptação ao uso do AASI, com consequente diminuição da evasão ao tratamento para a reabilitação auditiva de pacientes com perda auditiva.

\section{CONCLUSÃO}

O grupo que utilizou o site responsivo como ferramenta complementar ao processo de adaptação do AASI por usuários do SUS mostrou, a partir da avaliação da satisfação do usuário por meio do questionário SADL e dos dados da ferramenta datalogging, maior satisfação e maior tempo de uso diário do AASI.

\section{AGRADECIMENTOS}

YAC web.works (yac.com.br) pelo desenvolvimento do site responsivo.

\section{REFERÊNCIAS}

1. Beier LO, Pedroso F, Costa-Ferreira MID. Benefícios do treinamento auditivo em usuários de aparelho de amplificação Sonora individual: revisão sistemática. Rev CEFAC. 2015;17(4):1327-32. http://dx.doi. org/10.1590/1982-0216201517422614.

2. Signor RCF, Vieira SK, Berberian AP, Santana AP. Distúrbio de processamento auditivo $\mathrm{x}$ dificuldade de leitura e escrita: há uma relação? Rev Bras Lingüíst Apl. 2018;18(3):581-607. http://dx.doi. org/10.1590/1984-6398201813079.

3. Silva GBR, Almeida PL. Próteses auditivas por idosos: aspectos psicossociais, adaptação e qualidade de vida. Interações. Estud Pesqui Psicol. 2016;17(3):463-74.

4. Sales CB, Resende LM, Amaral CFS. Reabilitação auditiva em adultos: resultados de um programa de treinamento. Rev CEFAC. 2019;21(5):1-12.

5. Youssef BC, Mendes BCA, Costa EC, Ficker LB, Novaes BCAC. Effectiveness in adherence to auditory rehabilitation in children: family adherence group and initial therapy. Disturb Comum. 2017;29(4):73448.

6. Mazzarotto IHEK, Gonçalves CGO, Bellia CGL, Moretti CAM, Iantas MR. Integralidade do cuidado na atenção à saúde auditiva do adulto no SUS: acesso à reabilitação. Audiol Commun Res. 2019;24:1-8. http://dx.doi.org/10.1590/2317-6431-2018-2009.

7. Oliveira ARF, Alencar MSM. The use of health applications for mobile devices as sources of information and education in healthcare. Rev Digit Bibliotecon Cienc Inf. 2017;15(1):234-45.

8. Instituto Brasileiro de Geografia e Estatística. PNAD Contínua TIC 2017: internet chega a três em cada quatro domicílios do país. Agência IBGE [Internet]; $20 \mathrm{dez} 2018$ [citado em 2020 Out 9]. Disponível em: https://agenciadenoticias.ibge.gov.br/agencia-sala-de-imprensa/2013agencia-de-noticias/releases/23445-pnad-continua-tic-2017-internetchega-a-tres-em-cada-quatro-domicilios-do-pais

9. Galindo NNM, Sá GGM, Barbosa LU, Pereira JCN. Henriques AHBs, Barros LM. COVID-19 e tecnologia digital: aplicativos móveis disponíveis para download em smartphones. Texto Contexto Enferm. 2020;29:1-11.

10. Mondelli MFCG, Magalhães FF, Lauris JRP. Cultural adaptation of the SADL (Satisfaction with Amplification in Daily Life) questionaire for Brazilian Portuguese. Rev Bras Otorrinolaringol (Engl Ed). 2011;77(5):563-72.

11. Reis LR, Donato M, Sousa R, Escada P. Tradução, Adaptação Cultural e Validação da Escala Satisfaction with Amplification in Daily Life para o Português de Portugal. Acta Med Port. 2017;30(2):115-21. http://dx.doi.org/10.20344/amp.7794. PMid:28527478.

12. Cox RM, Alexander GC. Measuring satisfaction with amplification in daily life: the SADL scale. Ear Hear. 1999;20(4):306-20. http:// dx.doi.org/10.1097/00003446-199908000-00004. PMid:10466567. 
13. Silva DCO, Scharlach RC. Satisfação de usuários de aparelho de amplificação sonora individual atendidos em um centro auditivo. Rev CEFAC. 2015;17(6):1863-73. http://dx.doi.org/10.1590/1982021620151766115 .

14. Bevilacqua MC, Melo TM, Morettin M, Reis ACMB, Martinez MANS. Falhas técnicas dos aparelhos de amplificação sonora individual dispensados pelo Sistema Único de Saúde. Rev CEFAC. 2014;16(1):55-64. http://dx.doi.org/10.1590/1982-0216201411312.

15. Fernandes NF, Goffi-Gomez MVS, Magalhães ATM, Tsuji RK, Brito RV, Bento RF. Satisfação e qualidade de vida em usuários de implante auditivo de tronco cerebral. CoDAS. 2017;29(2):e20160059. http://dx.doi.org/10.1590/23171782/20172016059. PMid:28355382.

16. Dell'Antônia SF, Yudi Ikino CM, Carreirão W Fo. Grau de satisfação em usuários de próteses auditivas em um serviço de alta complexidade. Rev Bras Otorrinolaringol. 2013;79(5):555-63.

17. Solheim J, Hickson L. Hearing aid use in the elderly as measured by datalogging and self-report. Int J Audiol. 2017;56(7):472-9. http:// dx.doi.org/10.1080/14992027.2017.1303201. PMid:28332420.

18. Aazh H, Moore MCJ. Audiological rehabilitation for facilitating hearing aid use: a review. J Am Acad Audiol. 2017;28(3):248-60. http://dx.doi.org/10.3766/jaaa.16035. PMid:28277215. 
Anexo 1. Versão em português brasileiro do questionário Satisfaction With Amplification in Daily Life adaptado(15)

\section{SATISFAÇÃO COM AMPLIFICAÇÃO NA VIDA DIÁRIA}

Nome:

Data de Hoje

Data de Nascimento

INSTRUÇÕES: As questões listadas abaixo se referem as suas opiniões sobre o seu aparelho auditivo. Para cada questão, por favor, circule a letra que responde melhor a questão para você. A lista à direita fornece o significado de cada letra. Lembre-se que suas respostas devem mostrar suas opiniões gerais sobre o aparelho auditivo que você está usando atualmente ou que tem usado mais recentemente.

\section{A Não B Muito pouco C Pouco D Médio E Às vezes F Quase sempre G Sempre}

1. O seu aparelho auditivo the ajuda a entender as pessoas com as quais você fala quando comparado à época que não usava aparelho auditivo?
A B C D E F G

2. Você fica frustrado quando o seu aparelho auditivo capta sons que lhe impedem de ouvir o que você quer?

A B C D E F G

3. Você está convencido de que a obtenção de seu aparelho auditivo fazia parte dos seus maiores interesses?

A B C D E F G

4. Você acha que as pessoas percebem mais a sua perda auditiva quando você usa o aparelho?

A B C D E F G

5. O seu aparelho auditivo reduz o número de vezes que você tem que pedir para as pessoas repetirem?

A B C D E F G

6. Você acha que vale a pena usar o aparelho auditivo?

A B C D E F G

7. Você se sente incomodado quando necessita aumentar o volume e ocorre a microfonia?

A B C D E F G

8. Você está contente com a aparência do seu aparelho auditivo?

A B C D E F G

9. O uso do seu aparelho auditivo melhora a sua autoconfiança?

A B C D E F G

10. O som do seu aparelho auditivo é natural?

A B C D E F G

11. O seu aparelho auditivo é útil na MAIORIA dos telefones sem amplificador ou caixas de som?

A B C D E F G (Se você ouve bem ao telefone sem o aparelho, marque aqui)

12. A pessoa que lhe forneceu o aparelho auditivo era competente?

A B C D E F G

13. Você acha que usar o aparelho lhe faz parecer menos capacitado?

A B C D E F G

14. O custo do seu aparelho auditivo parece razoável para você?

A B C D E F G

15. Você está satisfeito com a frequência com a qual seu aparelho auditivo precisa de reparos?

A B C D E F G 